\title{
Lumen apposing metal stents in drainage of pancreatic walled-off necrosis, are they any better than plastic stents? A systematic review and meta-analysis of studies published since the revised Atlanta classification of pancreatic fluid collections
}

\author{
Babu P. Mohan, Mahendran Jayaraj ${ }^{1}$, Ravishankar Asokkumar' ${ }^{2}$ Mohammed Shakhatreh3, Parul Pahal, \\ Suresh Ponnada4, Udayakumar Navaneethan ${ }^{5}$, Douglas G. Adler ${ }^{6}$ \\ Department of Inpatient Medicine, University of Arizona, Banner University Medical Center, Tucson, Arizona, ${ }^{1}$ Division \\ of Gastroenterology/ Hepatology, University of Nevada, Las Vegas, ${ }^{3}$ Department of Hospital Medicine, Rapides Regional \\ Medical Center, Alexandria, Louisiana, ${ }^{4}$ Department of Hospital Medicine, Carilion Roanoke Memorial Hospital, Roanoke, \\ Virginia, ${ }^{5}$ Center for Interventional Endoscopy, Florida Hospital, Orlando, Florida, ${ }^{6}$ Division of Gastroenterology and \\ Hepatology, University of Utah School of Medicine, Salt Lake City, Utah, USA; ${ }^{2}$ Division of Gastroenterology and Hepatology, \\ Singapore General Hospital, Singapore
}

\section{ABSTRACT}

Lumen-apposing metal stents (LAMS) are increasingly being used in the drainage of pancreatic walled-off necrosis (WON). Best choice of stent is subject to argument, and studies are varied in the reported outcomes between LAMS and plastic stents (PS) to this end. We conducted a comprehensive search of multiple electronic databases and conference proceedings including PubMed, EMBASE, and Web of Science databases (earliest inception through July 2018) to identify studies that reported on the use of LAMS and PS in WON drainage. Studies published since the release of the revised Atlanta classification for pancreatic fluid collections (2014 to current) were included in the analysis. The outcomes were to estimate and compare the pooled rates of clinical success, and adverse-events. A total of 9 studies (737 patients) for LAMS and 6 studies (527 patients) for PS were included in the analysis. The pooled rate of clinical-success with LAMS was 88.5\% $\left(95 \%\right.$ CI 82.5-92.6, $\left.I^{2}=71.7\right)$ and with PS was $88.1 \%(95 \%$ CI 80.5-93.0, $P=78.1)$ and the difference was not statistically significant, $P=0.93$. No difference was noted in the pooled rates of all adverse-events, LAMS: $11.2 \%(6.8-17.9, P=82.0)$; vs PS: $15.9 \%(8.4-27.8, P=78.8) ; P=0.38$. Based on our meta-analysis, LAMS and PS demonstrate equal clinical outcomes and equal adverse-events in the drainage of pancreatic WON.

Key words: Lumen apposing metal stents, pancreatic, plastic stents, walled-off necrosis

\section{INTRODUCTION}

Pancreatic walled-off necrosis (WON) is defined by the revised Atlanta classification as "a collection that has

\begin{tabular}{|l|l|}
\hline \multicolumn{2}{|c|}{ Access this article online } \\
\hline Quick Response Code: & Website: \\
\hline
\end{tabular}

This is an open access journal, and articles are distributed under the terms of the Creative Commons Attribution-NonCommercial-ShareAlike 4.0 License, which allows others to remix, tweak, and build upon the work non-commercially, as long as appropriate credit is given and the new creations are licensed under the identical terms.

For reprints contact: reprints@medknow.com

How to cite this article: Mohan BP, Jayaraj M, Asokkumar R, Shakhatreh M, Pahal P, Ponnada S, et al. Lumen apposing metal stents in drainage of pancreatic walled-off necrosis, are they any better than plastic stents? A systematic review and meta-analysis of studies published since the revised Atlanta classification of pancreatic fluid collections. Endosc Ultrasound 2019;8:82-90.

\section{Address for correspondence}

Dr. Douglas G. Adler, Division of Gastroenterology and Hepatology, University of Utah School of Medicine, Salt Lake City, Utah, US. E-mail: douglas.adler@hsc.utah.edu

Received: 2018-09-14; Accepted: 2019-02-14; Published online: 2019-04-12 
occurred over time (usually at or after 4 weeks), when the necrotic collection matures and develops a thick nonepithelialized wall with visible debris or necrosis on imaging or ultrasound."[1] Among the available options to drain a WON, endoscopic ultrasound (EUS) guided drainage has become popular due to its speed and ease of use, lower morbidity and/or mortality as compared to surgical or percutaneous methods..$^{[2,3]}$

In EUS-guided drainage of WON, the endoscopist has the option of using either metal stents (MS) or plastic stents (PS) for maintaining the transluminal drainage. In recent years, lumen-apposing metal stents (LAMS) have been increasingly being used to this end. The results are inconsistent as to the performance of LAMS when compared to PS in the drainage of WON, and there is disagreement as to which stent is best in treating patients with WON. ${ }^{[4-7]}$ Bazerbachi et al. showed better clinical outcomes with LAMS when compared to PS $(91.5 \%$ vs. $80.9 \%$, Odds Ratio: 2.5, Confidence interval $(\mathrm{CI})$ : $1.4-4.3, P=0.001) \cdot{ }^{[8]}$ On the other hand, results from a randomized trial by Bang et al. reported no difference in the outcomes between LAMS and PS in the treatment of WON $(93.5 \%$ vs. $96.6 \%, P=0.99) .{ }^{\left[{ }^{[9]}\right.}$

Despite the available literature, there is no consensus yet on the best choice of stent when performing endoscopic drainage of WON. We conducted this meta-analysis to directly compare the clinical outcomes of LAMS and PS in the drainage of WON, from high quality studies published since the revised Atlanta classification of pancreatic fluid collections, and help address and potentially resolve this dilemma.

\section{METHODS}

\section{Search strategy}

We conducted a comprehensive search of several databases and conference proceedings, including PubMed, EMBASE, and Web of Science databases (earliest inception to July 2018). We followed the Preferred Reporting items for Systematic Reviews and Meta-Analyses guidelines, ${ }^{[10]}$ by using predefined protocol, to identify studies reporting the use of LAMS and PS in the drainage of WON. An experienced medical librarian using inputs from the study authors helped with the literature search.

Key words used in search included a combination of "Pancreatic necrosis," "walled off necrosis," "EUS guided pancreatic drainage," "LAMS in WON drainage,"
"Drainage of WON," "plastic stents in WON drainage," and "Pancreatic cyst drainage." The search was restricted to studies in human subjects and published in English language in peer-reviewed journals. Three authors (M.B., P.P., S.M.) independently reviewed the title and abstract of studies identified in primary search and excluded studies that did not address the research question, based on pre-specified exclusion and inclusion criteria. The full text of remaining articles was reviewed to determine whether it contained relevant information. Any discrepancy in article selection was resolved by consensus, and in discussion with a co-author (N.U.).

The bibliographic section of the selected articles, as well as the systematic and narrative articles on the topic was manually searched for additional relevant articles.

\section{Study selection}

In this meta-analysis, we included studies that met the following criteria: (1) use of LAMS in drainage of WON, (2) use of PS in drainage of WON, (3) studies with a minimum of 40 patients in study group, and (5) studies published in and/or after 2014. Studies irrespective of the study design, inpatient/outpatient setting, geography, and abstract/manuscript status, were included as long as they provided data needed for the analysis.

Following were our exclusion criteria: (1) studies published before 2014, (2) studies with less than 40 patients in the study group, (3) studies that primarily used a "dual-modality" treatment option that included both endoscopic and percutaneous drainage, (4) studies that were unclear on the reported clinical success rates, and (5) studies done in pediatric population (age $<18$ years).

In case of multiple publications from the same cohort, data from the most recent and/or most appropriate comprehensive report were included. In our search process, we encountered one such study by Jagielski et al. ${ }^{[1]}$

\section{Data abstraction and quality assessment}

Data on study-related outcomes in the individual studies were abstracted onto a standardized form by at least two authors (M.B., P.S.) independently, and three authors (M.B., S.M., A.R.) did the quality scoring independently.

In the situation of randomized trials and case-control studies, the data collection was done as number 
Mohan, et al.: versus plastic stents for pancreatic WON

of reported events $(n)$ out of total number of patients $(N)$ from each study. The collected data were treated akin to cohort studies, and therefore, we used a scale modified from the Newcastle-Ottawa scale for cohort studies to assess the quality of studies. ${ }^{[12]}$ This quality score consisted of seven questions: representative of the average adult in the community (1 point for population-based studies, 0.5 point for multi-center studies, 0 point for a single-center hospital-based study); cohort size (1 point for $>40$ patients); information on clinical success ( 1 point if reported, 0.5 point if not reported and had to be derived, 0 point if not reported); information on adverse-events ( 1 point if reported, 0 point if not reported); information on adverse-events subtypes (1 point if reported, 0 point if not reported); type of article write-up (1 point if original manuscript, 0.5 point if abstract); attrition rate ( 1 point if all patients were accounted for, 0.5 point if $<50 \%$ of patients lost to follow-up, 0 point if $>50 \%$ of patients lost to follow-up). A score of $\geq 6,4-5$, and $\leq 3$ was considered suggestive of high-quality, medium-quality, and low-quality study, respectively.

\section{Outcomes assessed}

The primary analysis focused on calculating the pooled rates of clinical success with LAMS and PS in the drainage of pancreatic WON.

Our secondary analysis focused on the analysis of adverse-events and its subtypes with LAMS and PS. The adverse-events subtypes analyzed were bleeding, sepsis, perforation, and stent migration.

Assessment methodology and definitions

The collected data were matched between the LAMS and PS groups. The timing of conducting the study, baseline patient characteristics, etiologies of WON, mean and/or median WON size, and endoscopic techniques used were comparable between the groups. We, therefore, statistically compared the calculated outcomes between LAMS and PS. Although, this model of comparison is considered statistically weak when compared to a randomized-controlled trial, the approach is comparable to a retrospective case-control study with matched groups. ${ }^{[13]}$

In the included studies, clinical success was either defined as the resolution of clinical symptoms along with reduction of WON size to $>50 \%$ on imaging in 4-8 weeks; or as a decrease in size to $\leq 2 \mathrm{~cm}$ on imaging, without the need for the placement of an additional endoscopic or percutaneous stent or drain, or surgery; disappearance of symptoms or inflammation regardless of the collection size.

Adverse-events were defined as complications that required hospital admission and/or endoscopic or surgical intervention. Sepsis was defined as new event of infection after the initial endoscopic drainage, as proven by new-onset fever and/or positive blood cultures. Stent migration was defined, as a complication if an intervention was required to retrieve the stent either from the WON cavity or from the enteral lumen. Perforation was defined either of the WON and/or the adjacent bowel lumen, with free-air in peritoneum and peritoneal signs.

\section{Statistical analysis}

We used meta-analysis techniques to calculate the pooled estimates in each case following the methods suggested by DerSimonian and Laird using the random-effects model and our application can be seen to fit within their general approach (where effect is measured by probability of risk). ${ }^{[14]}$ When the incidence of an outcome was zero in a study, a correction of 0.01 was added to the number of incident cases before statistical analysis. ${ }^{[15]}$ We assessed heterogeneity between study-specific estimates by using Cochran $\mathrm{Q}$ statistical test for heterogeneity and the $I^{2}$ statistics. ${ }^{[16,17]}$ In this, values of $<30 \%, 30 \%-60 \%$, $61 \%-75 \%$, and $>75 \%$ were suggestive of low, moderate, substantial, and considerable heterogeneity, respectively. ${ }^{[18]}$ Since random-effects model estimates an average effect, we calculated the $95 \%$ of prediction interval, which deals with the dispersion of the effects. ${ }^{[19,20]}$ Publication bias was ascertained, qualitatively, by visual inspection of funnel plot and quantitatively, by the Egger test. ${ }^{[21]}$ When publication bias was present, further statistics using the fail-Safe $\mathrm{N}$ test and Duval and Tweedie's "Trim and Fill" test was used to ascertain the impact of the bias. ${ }^{[2]}$ Three levels of impact were reported based on the concordance between the reported results and the actual estimate if there were no bias. The impact was reported as minimal if both versions were estimated to be same, modest if effect size changed substantially, but the final finding would still remain the same, and severe if basic final conclusion of the analysis is threatened by the bias. ${ }^{[23]}$

All analyses were performed using the Comprehensive Meta-Analysis software, version 3 (BioStat, Englewood, NJ). 
Mohan, et al:: versus plastic stents for pancreatic WON

\section{RESULTS}

\section{Search results and population characteristics}

From an initial total of 1052 citations identified using our search strategy, our screening and eligibility check resulted in 58 relevant records. Twenty-one studies were removed due to $<40$ sample-size. Four studies were removed due to publication year before 2014. A total of 15 studies (1264 patients) were included in the final analysis of which LAMS was used in nine studies with 737 patients, ${ }^{[4,5,24-30]}$ and PS was used in six studies with 527 patients. ${ }^{[4,11,29,31-33]}$

The schematic diagram of study selection is illustrated in Figure 1.

Baseline population characteristics were comparable in LAMS and PS groups. The mean and/or median age was from 35 years to 60 years, with predominantly male population (range 60\% to $90 \%$ ). Mean WON size was in the range of $95 \mathrm{~mm}-121 \mathrm{~mm}$ in both groups. Access to WON was via the transgastric route in $88 \%$ of the time, and the rest were via a trans-duodenal approach. Median number of procedures to achieve clinical success ranged from 1.5 to 2.8 in LAMS group and 2.7 to 4.0 in PS group. The most frequent

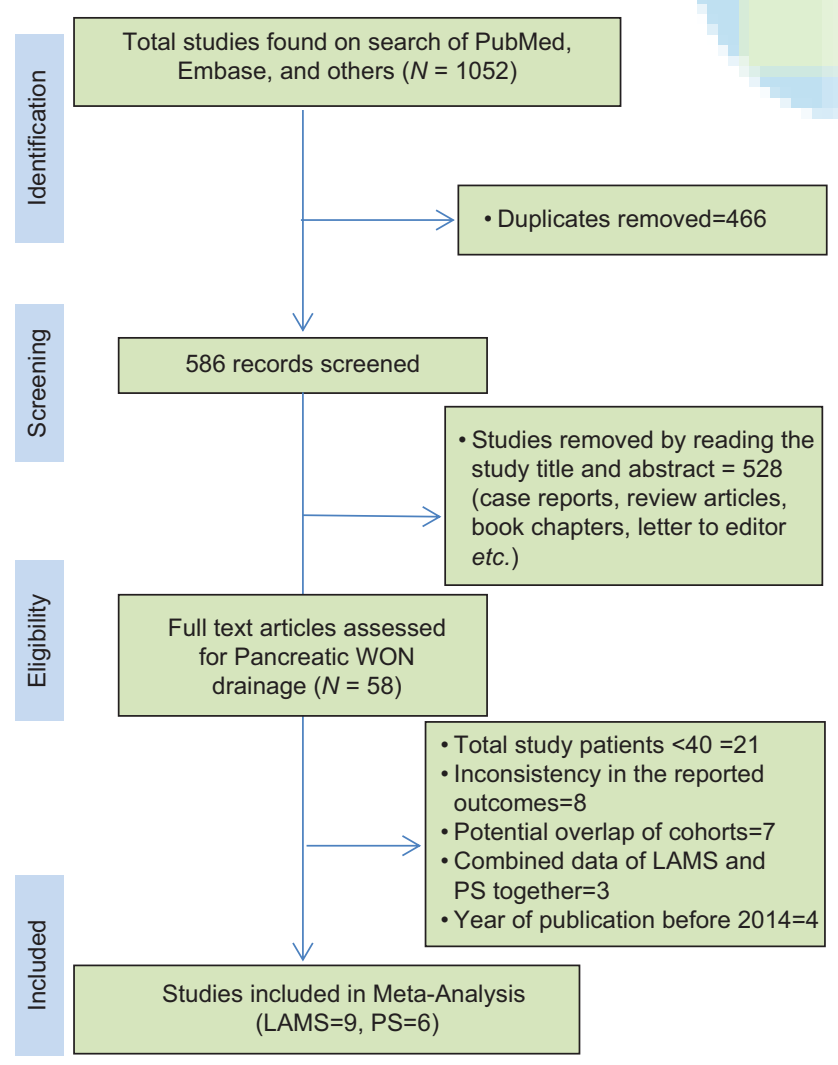

Figure 1. Study flow and selection. WON: Walled-off necrosis etiologies causing pancreatitis and evolution to WON were alcohol $(45 \%)$, gallstones $(37 \%)$, and idiopathic causes $(18 \%)$.

\section{Characteristics and quality of included studies}

Table 1 describes the characteristics of the included studies.

Out of the included 15 studies, none were population-based. Six studies ${ }^{[25-30]}$ were from multicenter data and rest were from single-center. All studies were chosen with minimum of 40 patients in the study group based on the inclusion and/or exclusion criteria. All studies reported adequately on the clinical-success outcomes, and all were original manuscripts with no significant attrition rate. All studies reported adequately on the adverse-events and its subtypes, except one. Overall, all studies were considered of high quality (Supplemental Table 1).

Etiology and clinical indication

The most frequent etiologies were alcohol, gallstones, and idiopathic causes. Clinical indication for drainage of WON was as follows: refractory abdominal pain; gastric outlet, or biliary obstruction; ongoing systemic illness, anorexia, weight loss; rapidly enlarging WON; and infected WON.

Recurrence was defined as an increase in collection size to $>3 \mathrm{~cm}$ or relapse of symptoms during a 6-month follow-up.

Procedure description with use of lumen-apposing metal stents

LAMS used in the selected studies were as follows: Hot Axios ${ }^{[25,26]}$ and Axios ${ }^{[5,25,27-30]}$ (Xlumena, Mountain View, California, USA). Nagi ${ }^{[4,24]}$ and Niti-S Spaxus $^{[5]}$ (Taewoong Medical, Seoul, South Korea). ${ }^{[24]}$ Hanaro $^{[5]}$ (MI Tech, Seoul, South Korea).

Procedures were performed with patients under deep sedation or general anesthesia. A prophylactic antibiotic was administered before procedure in majority of the studies. The WON collection was identified using a linear echoendoscope and accessed through the Seldinger technique, whereby a fine-needle aspiration is done followed by guide-wire insertion. The internal ostomy tract was then dilated using a balloon to facilitate passage of the stent delivery system over the wire. In case of a thick wall, an over-the-wire needle-knife catheter or $10 \mathrm{~F}$ cystotome 
Mohan, et al:: versus plastic stents for pancreatic WON

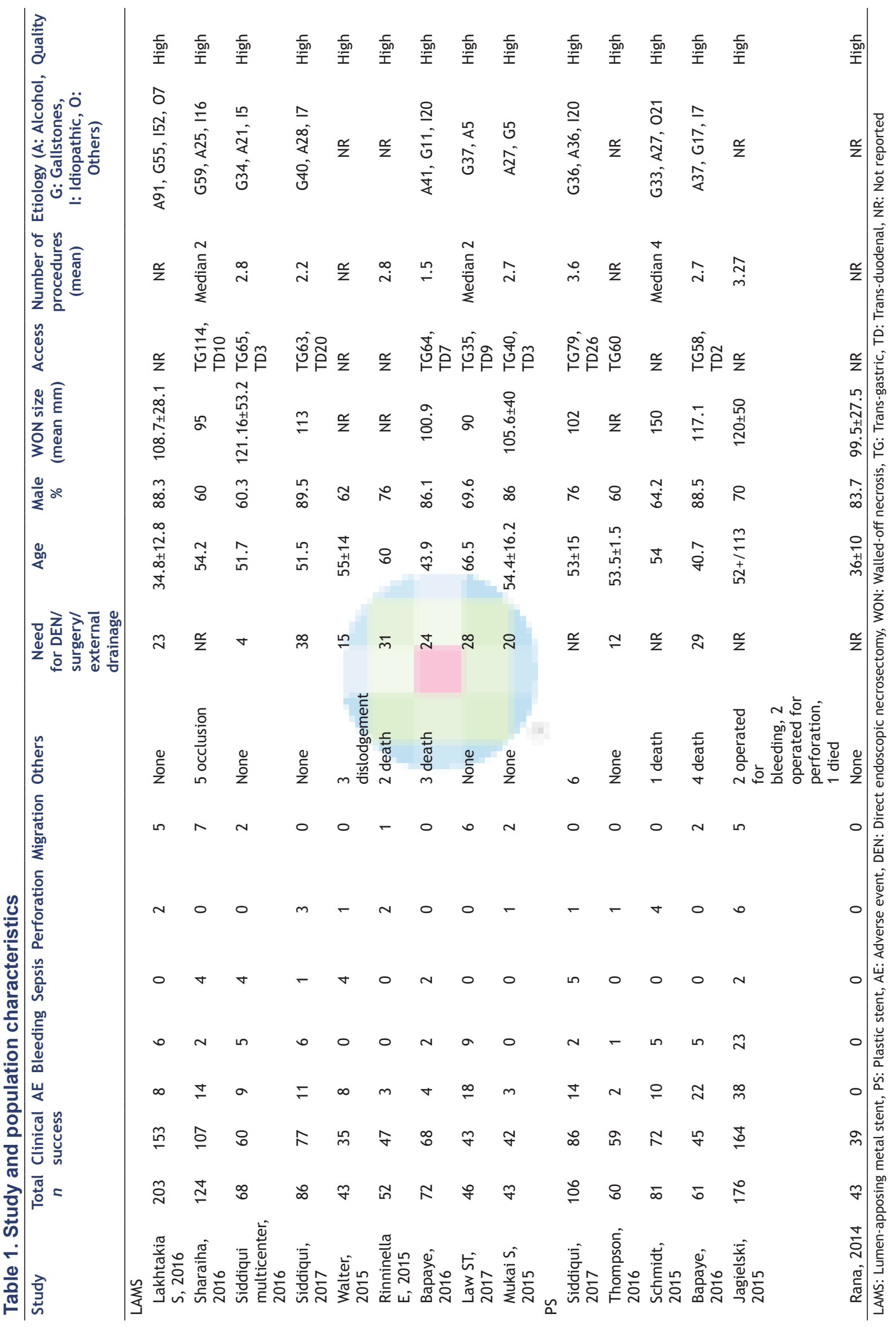


Mohan, et al.: versus plastic stents for pancreatic WON

(Cook Endoscopy, Winston-Salem, NC) was used to dissect the tract before balloon dilation. The LAMS delivery system was then advanced into the WON collection, and deployed under endosonographic, endoscopic, and fluoroscopic guidance. In cases where the Hot-Axios device was used, the WON was punctured using the electrocautery tip and the delivery catheter was introduced into the cavity followed by deployment of the stent.

Procedure description with use of plastic stents In majority of studies, the endoscopic intervention was under deep sedation. Double pigtail PS (7-Fr) were used. Fistula creation was performed on top of the largest bulge into the gastric or duodenal wall. After puncturing with a 19G needle, a guide-wire was coiled within the WON under fluoroscopic guidance. After sequential dilating the transmural tract using balloon dilators the PS were placed into the WON through the transmural fistula. The number of PS placed varied based on the endoscopist's decision and the size of WON.

\section{Clinical success of lumen-apposing metal stents and} plastic stents in drainage of walled-off necrosis

LAMS were used in nine studies with 737 patients, ${ }^{[4,5,24-30]}$ and PS were used in six studies with 527 patients. ${ }^{[4,11,28,31-33]}$ The pooled rate of clinical success with LAMS was $88.5 \%$ (95\% CI 82.5-92.6) and with PS was $88.1 \%$ (95\% CI 80.5-93.0). There was no statistical significance to the difference, $P=0.93$. Figure 2. Forest plot - Clinical success.

Substantial heterogeneity (LAMS: $I^{2}=71.7$ and PS: $\left.I^{2}=78.1\right)$ was noted, which was comparable in both groups. The calculated prediction interval with LAMS was $0.874(95 \%$ interval $-0.321-2.069$, range $=2.39)$, and with PS was $0.883(-0.734-2.50$, range $=3.23)$.

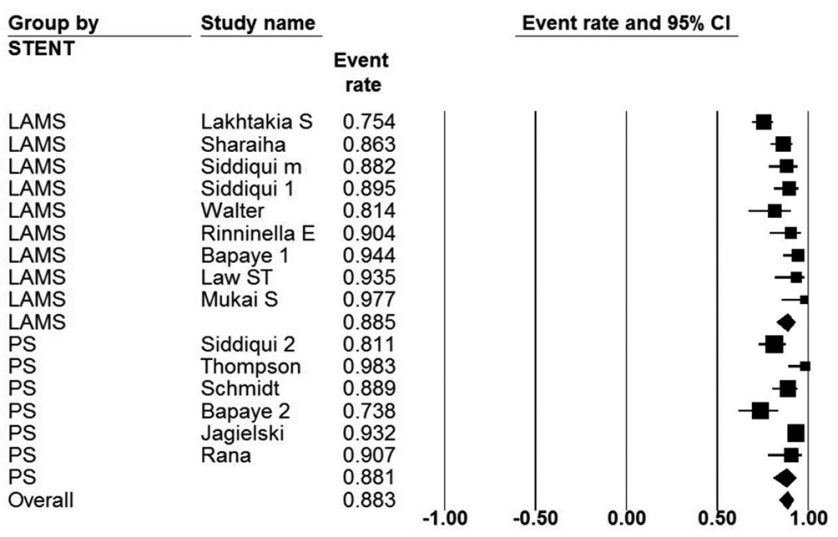

Figure 2. Forest plot - Clinical success

\section{Adverse events}

The pooled rate of all adverse-events with LAMS was $11.2 \%(95 \%$ CI $6.8-17.9)$ and with PS was $15.9 \%$ (95\% CI 8.4-27.8). There was no statistical significance to the difference, $P=0.38$ (Figure 3. Forest plot - all adverse events).

Substantial to considerable heterogeneity (LAMS: $I^{2}=82.0$, PS: $I^{2}=78.8$ ) was noted. The calculated prediction interval for the adverse-events with LAMS was 0.108 (95\% interval - 1.45-1.67, range 3.12) and with PS was 0.162 (95\% interval - 1.59-1.91, range 3.50 ).

The calculated pooled rates for bleeding, sepsis, perforation, and stent-migration are summarized in Table 2 (Forest plots: please see supplementary material).

\section{Validation of meta-analysis results}

Sensitivity analysis

To assess whether any one study had a dominant effect on the meta-analysis, we excluded one study at a time

Table 2. Results lumen-apposing metal stent versus plastic stent

\begin{tabular}{|c|c|c|c|}
\hline \multirow[t]{2}{*}{ Subgroup } & \multicolumn{2}{|c|}{$95 \% \mathrm{Cl}, I^{2}$} & \multirow[t]{2}{*}{$P$} \\
\hline & $\begin{array}{l}\text { LAMS (9studies, } \\
737 \text { patients) }\end{array}$ & $\begin{array}{l}\text { PS ( } 6 \text { studies, } \\
527 \text { patients) }\end{array}$ & \\
\hline $\begin{array}{l}\text { Clinical } \\
\text { success }\end{array}$ & $88.5 \%(82.5-92.6,71.7)$ & $88.1 \%(80.5-93.0,78.1)$ & 0.93 \\
\hline All AE & $11.2 \%(6.8-17.9,82.0)$ & $15.9 \%(8.4-27.8,78.8)$ & 0.38 \\
\hline Bleeding & $5.4 \%(2.7-10.5,63.7)$ & $5.9 \%(2.7-12.3,61.5)$ & 0.88 \\
\hline Sepsis & $4.5 \%(2.7-7.3,0)$ & $3.0 \%(1.5-6.2,0)$ & 0.38 \\
\hline Perforation & $2.4 \%(1.3-4.5,0)$ & $3.2 \%(1.8-5.6,0)$ & 0.49 \\
\hline $\begin{array}{l}\text { Stent } \\
\text { migration }\end{array}$ & $5.1 \%(3.4-7.5,27.5)$ & $2.9 \%(1.4-5.9,0)$ & 0.18 \\
\hline
\end{tabular}

Cl: Confidence interval, LAMS: Lumen-apposing metal stent, PS: Plastic stent, AE: Adverse event

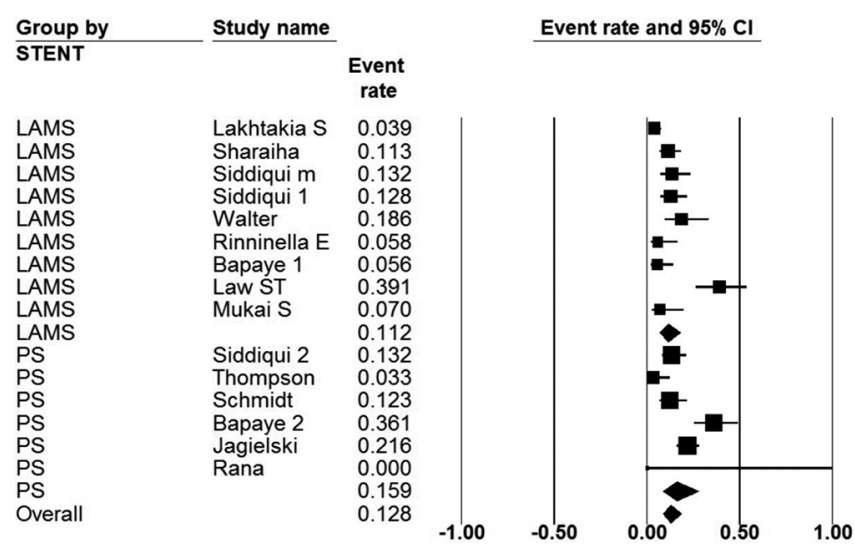

Figure 3. Forest plot - all adverse events 
Mohan, et al.: versus plastic stents for pancreatic WON

and analyzed its effect on the main summary estimate. On this analysis, no single study significantly affected the outcome or the heterogeneity.

\section{Publication bias}

Based on visual inspection of the funnel plot as well as quantitative measurement that used the Egger regression test, there was evidence of publication bias. There was likelihood that small, negative-outcome studies were not published. Further statistics using the fail-Safe $\mathrm{N}$ test and Duval and Tweedie's 'Trim and Fill' test reveal that the impact of the possible publication bias appears to be minimal and would not change the calculated estimate or the conclusion of this meta-analysis (S. Figure 5. Funnel plot).

\section{DISCUSSION}

The current available options to drain a WON are as follows: direct surgical drainage, endoscopy-guided drainage via stent placement with or without direct endoscopic necrosectomy, and percutaneous drainage, usually performed by interventional radiologists. Lumen-apposing metal stents (LAMS) with their larger diameter are postulated to help better drain the WON contents than the small caliber PS. ${ }^{[9,34]}$ LAMS once placed, by virtue of their large diameter, allows for passage of the endoscope into the necrotic cavity, which can then be used to lavage the necrotic cavity and dissect and remove the necrotic tissue. Multiple repeat DEN is possible with LAMS and negates the need to remove the stent each time as is the case with PS. Studies have shown a need for fewer repeat treatment sessions with LAMS as compared to PS. ${ }^{[6,9]}$ As a result, there is growing interest in using LAMS in the drainage of pancreatic WON. A recent cost-effectiveness analysis comparing LAMS with PS by Chen et al., reported LAMS to be more clinically efficacious than PS $(92 \%$ vs. $84 \%)$ in the successful drainage of WON, but with average cost per patient of US $\$ 20,029$ for LAMS and US\$15,941 for PS. ${ }^{[7]}$

Our study is the first meta-analysis to primarily compare the clinical outcomes of LAMS and PS in the drainage of WON, from good quality studies published since the revised Atlanta classification of pancreatic fluid collections. Based on our analysis, we report no difference between LAMS and PS in the clinical success of pancreatic WON drainage $88.5 \%$ with LAMS $v$ s. $88.1 \%$ with PS, $P=0.93$ ), and our results are on par with Bang et al.'s ${ }^{[0]}$ randomized trial. Resolution with LAMS was achieved with lesser mean number of procedures (mean 1.5-2.8) as compared to PS (mean 2.7-4.0).

The other meta-analysis by Bazerbachi et al. ${ }^{[8]}$ primarily compared MS to PS in the drainage of WON and then a subsequent subgroup analysis compared the outcomes of LAMS to PS. The pooled results were derived from five studies that originally compared LAMS and PS in the drainage of WON. The study differs in the primary outcome and the methodology of the comparison. We extracted data for LAMS and PS from comparable individual studies, and matched the groups for basic study characteristics and compared the reported outcomes. The favorable results to LAMS, reported in the meta-analysis by Bazerbachi et al. ${ }^{[8]}$ might have been influenced by inclusion of smaller studies and studies from different time-line between the groups. Small sized studies add to the heterogeneity and negatively affect the overall pooled results. ${ }^{[3]}$

In our analysis of adverse-events, the rates for all adverse-events and the rates for bleeding, sepsis, perforation, and stent-migration were similar between LAMS and PS. In their randomized-trial, Bang et al. ${ }^{[9]}$ noticed a high rate of adverse-events with LAMS due to which they had to change their study protocol midway, and proposed to get a CT scan check after 3 weeks. They reported three cases of bleeding and 10 cases of overall adverse-events out of their 30 patients who were treated with LAMS. The rates were, however, similar in both groups after change of protocol. Although not statistically significant, we did note a numerically higher rate for stent-migration with LAMS $(5.1 \%)$ as compared to PS $(2.9 \%), P=0.18$. In our collected data, death was reported in five patients from the LAMS group and six patients in PS group. We were not able to calculate the pooled rate for death due to paucity in the number of studies that they were reported from.

We limited our study selection to those published on or after 2014. There are several reasons to this. One, LAMS were clinically introduced to the US market in 2014. Two, the revised Atlanta classification for pancreatic WON was first published online on October 25, 2012 and printed in early 2013.[1] Therefore, the authors agreed to compare the outcomes of LAMS and PS in WON drainage, from studies done during similar time frame. By doing this, we hoped to match both study groups in the techniques used, and avoid potential 
Mohan, et al:: versus plastic stents for pancreatic WON

variability due to previously used diagnostic criteria, procedural techniques and instruments. Although including all available studies in the literature would increase the total patients available for the meta-analysis, this would in fact negatively impact the strength of the evidence if included studies were of small sample size. ${ }^{[13,35]}$

The strengths of this review are as follows: systematic literature search with well-defined inclusion criteria, uniform usage of the revised Atlanta classification in the definition of WON, carefully excluding redundant studies, inclusion of good quality studies published from 2014 to current, detailed extraction of data with rigorous evaluation of study quality, matching basic patient and study characteristics between the study groups, and statistics to establish and/or refute the validity of the results of our meta-analysis.

There were limitations to this study. We used an indirect comparison strategy for the outcomes with LAMS and PS. The included studies were not representative of the general population and community practice, with most studies being performed in the tertiary-care referral centers. Considerable heterogeneity was noted in our analysis along with wide range to prediction-intervals, which could have been due to multiple reasons and they are as follows: differences in endoscopic techniques between centers, operator dependent variability, variability in the number of endoscopic sessions to resolution of WON, variability in the use of naso-cystic drains, variability in the route of access to WON, variability on the usage of "multi-gate" drainage, and variability in the grade of necrosis and/or solid components in the WON. We were not able to assess the outcomes based on these important parameters. Although, we were able to adjust for most of these parameters between the LAMS and PS groups, variability within the group still existed. We were not able to identify and classify adverse-events based on their severity. We were not able to assess the impact of disconnected pancreatic duct syndrome (DPDS) on the assessed outcomes. DPDS is often overlooked, yet important complication of WON and permanently placed in situ PS are preferred in such circumstances. ${ }^{[36]}$

\section{CONCLUSION}

Our meta-analysis demonstrates essentially equal clinical outcomes between LAMS and PS in terms of successful drainage and adverse-events in the endoscopic drainage of pancreatic WON. LAMS are easier to use for performing DEN, may reduce the total number of procedures, but come at increased cost.

\section{Supplementary Materials}

Supplementary information is linked to the online version of the paper on the Endoscopic Ultrasound website.

\section{Financial support and sponsorship}

Nil.

\section{Conflicts of interest}

There are no conflicts of interest.

\section{REFERENCES}

1. Banks PA, Bollen TL, Dervenis C, et al. Classification of acute pancreatitis--2012: Revision of the Atlanta classification and definitions by international consensus. Gut 2013;62:102-11.

2. Keane MG, Sze SF, Cieplik N, et al. Endoscopic versus percutaneous drainage of symptomatic pancreatic fluid collections: A 14-year experience from a tertiary hepatobiliary centre. Surg Endosc 2016;30:3730-40.

3. van Brunschot S, van Grinsven J, van Santvoort HC, et al. Endoscopic or surgical step-up approach for infected necrotising pancreatitis: A multicentre randomised trial. Lancet 2018;391:51-8.

4. Bapaye A, Dubale NA, Sheth KA, et al. Endoscopic ultrasonography-guided transmural drainage of walled-off pancreatic necrosis: Comparison between a specially designed fully covered bi-flanged metal stent and multiple plastic stents. Dig Endosc 2017;29:104-10.

5. Mukai S, Itoi T, Baron TH, et al. Endoscopic ultrasound-guided placement of plastic vs. biflanged metal stents for therapy of walled-off necrosis: A retrospective single-center series. Endoscopy 2015;47:47-55.

6. Abu Dayyeh BK, Mukewar S, Majumder S, et al. Large-caliber metal stents versus plastic stents for the management of pancreatic walled-off necrosis. Gastrointest Endosc 2018;87:141-9.

7. Chen YI, Barkun AN, Adam V, et al. Cost-effectiveness analysis comparing lumen-apposing metal stents with plastic stents in the management of pancreatic walled-off necrosis. Gastrointest Endosc 2018;88:267-76.e1.

8. Bazerbachi F, Sawas T, Vargas EJ, et al. Metal stents versus plastic stents for the management of pancreatic walled-off necrosis: A systematic review and meta-analysis. Gastrointest Endosc 2018;87:30-42.e15.

9. Bang JY, Navaneethan U, Hasan MK, et al. Non-superiority of lumen-apposing metal stents over plastic stents for drainage of walled-off necrosis in a randomised trial. Gut 2018. pii: gutjnl-2017-315335.

10. Moher D, Liberati A, Tetzlaff J, et al. Preferred reporting items for systematic reviews and meta-analyses: The PRISMA statement. Ann Intern Med 2009;151:264-9, W64.

11. Jagielski M, Smoczyński M, Jabłońska A, et al. The role of endoscopic ultrasonography in endoscopic debridement of walled-off pancreatic necrosis - A single center experience. Pancreatology 2015;15:503-7.

12. Stang A. Critical evaluation of the Newcastle-Ottawa scale for the assessment of the quality of nonrandomized studies in meta-analyses. Eur J Epidemiol 2010;25:603-5.

13. Thompson SG, Pocock SJ. Can meta-analyses be trusted? Lancet 1991;338:1127-30.

14. DerSimonian R, Laird N. Meta-analysis in clinical trials. Control Clin Trials 1986;7:177-88.

15. Sutton AJ, Abrams KR, Jones DR, et al. Methods for Meta-Analysis in 
Mohan, et al.: versus plastic stents for pancreatic WON

Medical Research. New York: John Wiley \& Sons Ltd., 2000. p. 205-28.

16. Kanwal F, White D. "Systematic reviews and meta-analyses" in clinical gastroenterology and hepatology. Clin Gastroenterol Hepatol 2012;10:1184-6.

17. Higgins JP, Thompson SG, Deeks JJ, et al. Measuring inconsistency in meta-analyses. BMJ 2003;327:557-60.

18. Guyatt GH, Oxman AD, Kunz R, et al. GRADE guidelines: 7. Rating the quality of evidence - Inconsistency. J Clin Epidemiol 2011;64:1294-302.

19. Higgins JP, Thompson SG, Spiegelhalter DJ. A re-evaluation of random-effects meta-analysis. J R Stat Soc Ser A Stat Soc 2009;172:137-59.

20. Riley RD, Higgins JP, Deeks JJ. Interpretation of random effects meta-analyses. BMJ 2011;342:d549.

21. Easterbrook PJ, Berlin JA, Gopalan R, et al. Publication bias in clinical research. Lancet 1991;337:867-72.

22. Duval S, Tweedie R. Trim and fill: A simple funnel-plot-based method of testing and adjusting for publication bias in meta-analysis. Biometrics 2000;56:455-63.

23. Rothstein HR, Sutton AJ, Borenstein M. Publication Bias in Meta-Analysis: Prevention, Assessment and Adjustments.New York: John Wiley \& Sons Ltd., 2006.

24. Lakhtakia S, Basha J, Talukdar R, et al. Endoscopic "step-up approach" using a dedicated biflanged metal stent reduces the need for direct necrosectomy in walled-off necrosis (with videos). Gastrointest Endosc 2017;85:1243-52

25. Law ST, De La SernaHiguera C, Simón PG, et al. Comparison of clinical efficacies and safeties of lumen-apposing metal stent and conventional-type metal stent-assisted EUS-guided pancreatic wall-off necrosis drainage: A real-life experience in a tertiary hospital. Surg Endosc 2018;32:2448-53.

26. Rinninella E, Kunda R, Dollhopf M, et al. EUS-guided drainage of pancreatic fluid collections using a novel lumen-apposing metal stent on an electrocautery-enhanced delivery system: A large retrospective study (with video). Gastrointest Endosc 2015;82:1039-46.

27. Sharaiha RZ, Tyberg A, Khashab MA, et al. Endoscopic therapy with lumen-apposing metal stents is safe and effective for patients with pancreatic walled-off necrosis. Clin Gastroenterol Hepatol 2016;14:1797-803

28. Siddiqui AA, Adler DG, Nieto J, et al. EUS-guided drainage of peripancreatic fluid collections and necrosis by using a novel lumen-apposing stent: A large retrospective, multicenter U.S. experience (with videos). Gastrointest Endosc 2016;83:699-707.

29. Siddiqui AA, Kowalski TE, Loren DE, et al. Fully covered self-expanding metal stents versus lumen-apposing fully covered self-expanding metal stent versus plastic stents for endoscopic drainage of pancreatic walled-off necrosis: Clinical outcomes and success. Gastrointest Endosc 2017;85:758-65.

30. Walter D, Will U, Sanchez-Yague A, et al. A novel lumen-apposing metal stent for endoscopic ultrasound-guided drainage of pancreatic fluid collections: A prospective cohort study. Endoscopy 2015;47:63-7.

31. Rana SS, Bhasin DK, Sharma RK, et al. Do the morphological features of walled off pancreatic necrosis on endoscopic ultrasound determine the outcome of endoscopic transmural drainage? Endosc Ultrasound 2014;3:118-22.

32. Schmidt PN, Novovic S, Roug S, et al. Endoscopic, transmural drainage and necrosectomy for walled-off pancreatic and peripancreatic necrosis is associated with low mortality - A single-center experience. Scand J Gastroenterol 2015;50:611-8.

33. Thompson CC, Kumar N, Slattery J, et al. A standardized method for endoscopic necrosectomy improves complication and mortality rates. Pancreatology 2016;16:66-72.

34. Varadarajulu S, Bang JY, Phadnis MA, et al. Endoscopic transmural drainage of peripancreatic fluid collections: Outcomes and predictors of treatment success in 211 consecutive patients. J Gastrointest Surg 2011;15:2080-8

35. IntHout J, Ioannidis JP, Borm GF, et al. Small studies are more heterogeneous than large ones: A meta-meta-analysis. J Clin Epidemiol 2015;68:860-9.

36. Bang JY, Wilcox CM, Navaneethan U, et al. Impact of disconnected pancreatic duct syndrome on the endoscopic management of pancreatic fluid collections. Ann Surg 2018;267:561-8. 


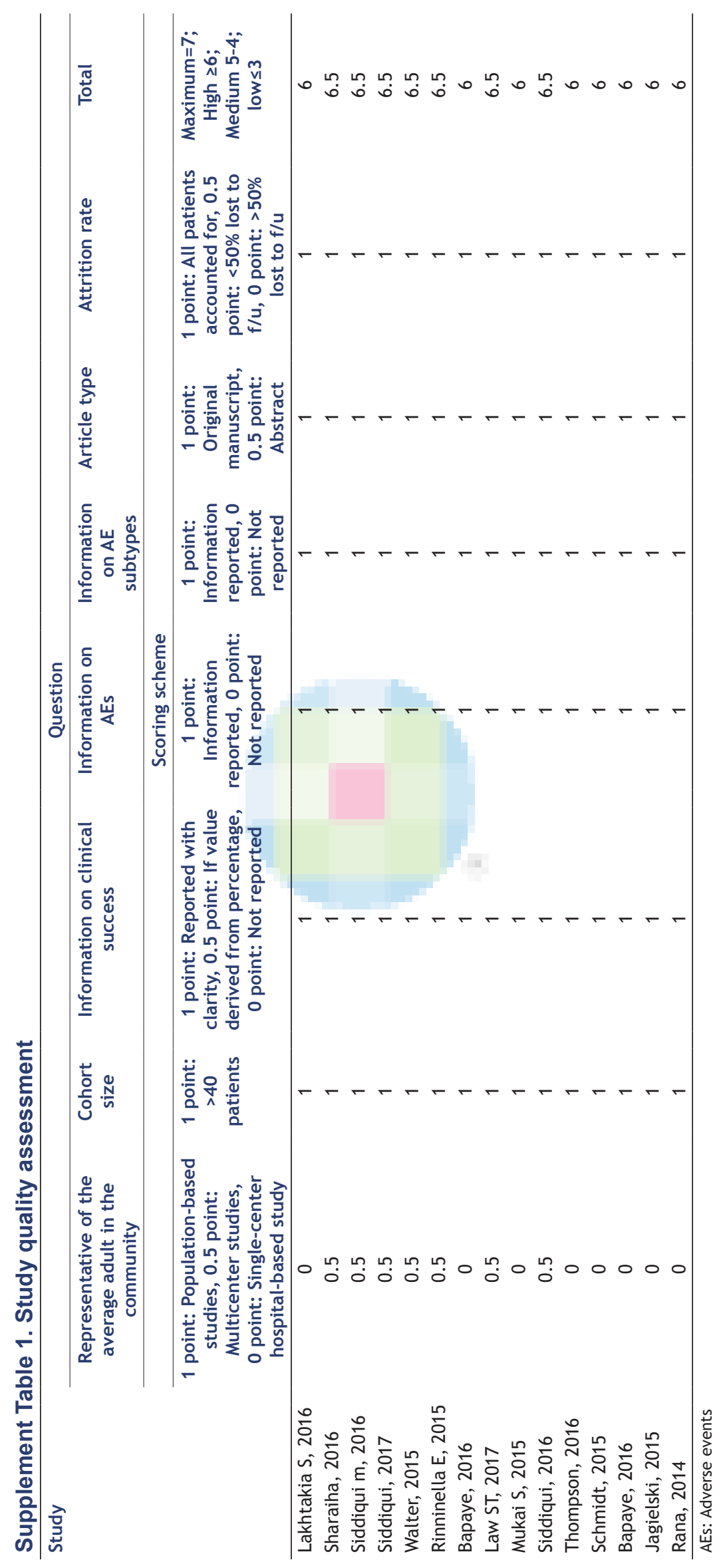




\section{Supplement Materials}
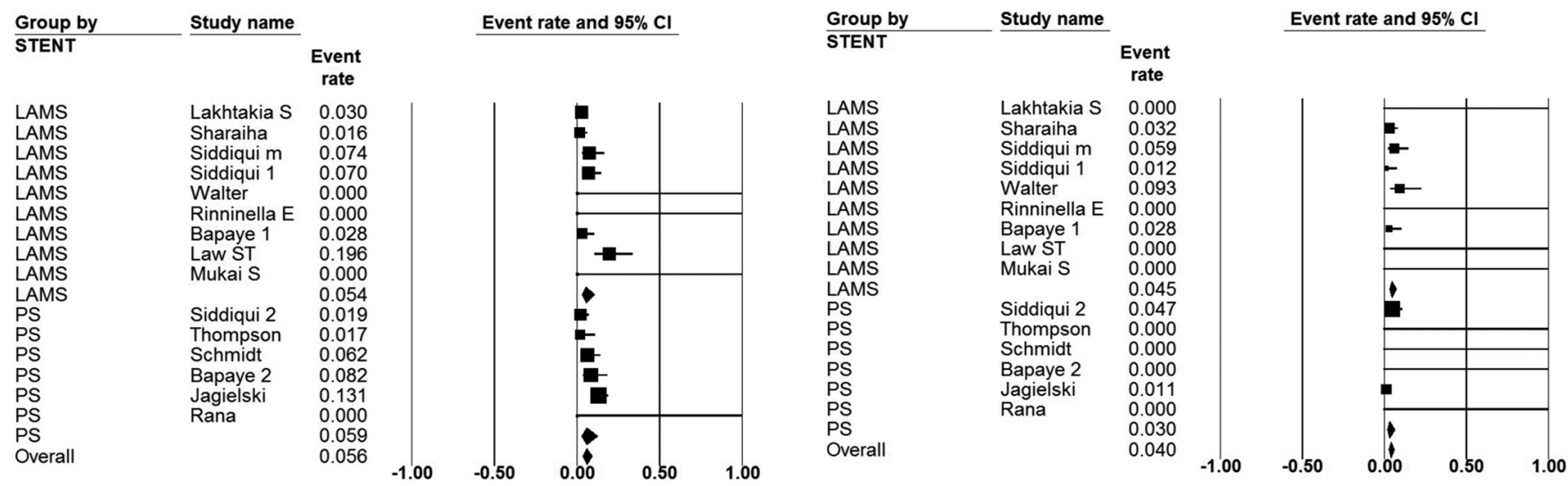

Supplement Figure 1. Forest plot - Bleeding

Supplement Figure 2. Forest plot - Sepsis

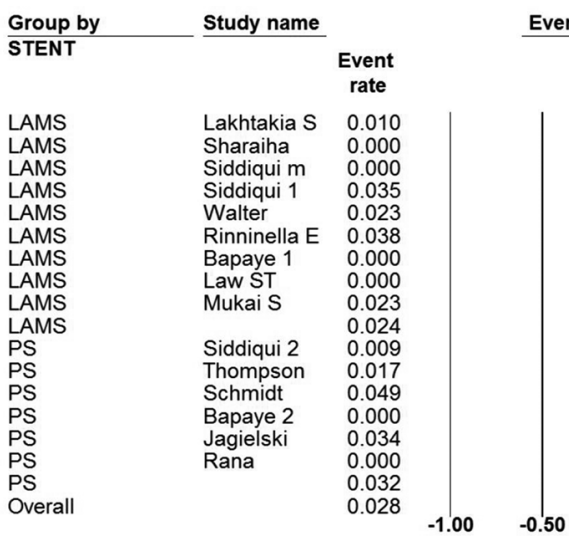

Event rate and $95 \% \mathrm{Cl}$

Group b
STENT
LAMS
LAMS
LAMS
LAMS
LAMS
LAMS
LAMS
LAMS
LAMS
LAMS
PS
PS
PS
PS
PS
PS
PS
Overall

Study name

Event rate and $95 \% \mathrm{Cl}$

$-0.50$

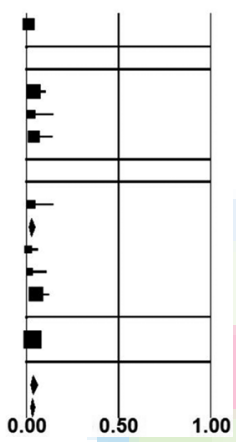

Supplement Figure 3. Forest plot - Perforation

Supplement Figure 4. Forest plot - Stent migration

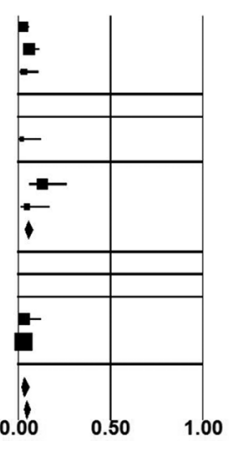

Funnel Plot of Standard Error by Logit event rate

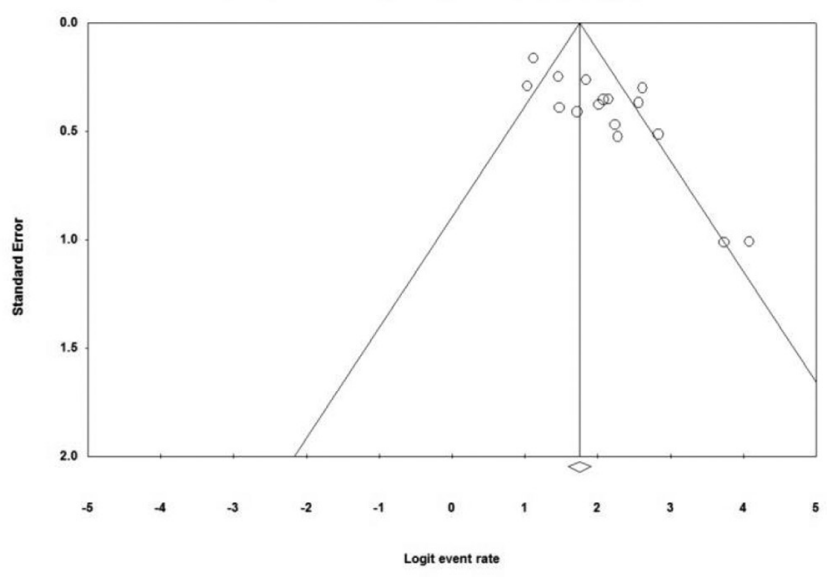

Supplement Figure 5. Funnel plot 